\title{
COMPTONIZATION AS BLAZAR HIGH ENERGY EMISSION
}

OLIVER DREISSIGACKER

Landessternwarte Heidelberg, Germany

http://www.lsw.uni-heidelberg.de/ odreissi/

\section{Summary}

We explain the overall continuous GrazAR (Gamma Ray Blazar) spectrum from the synchrotron turnover to the EGRET $\mathrm{GeV}$ detections by means of Comptonization in the parsec scale jet's substructures.

While making use of the constraints on the synchrotron spectrum and other measurable quantities, no exotic particle acceleration is needed to achieve the high energy output.

We show, that the "Lighthouse Model" of blobs of relativistic electrons, travelling with the jet plasma at relativistic speeds, produce both, correct timescales and shapes for the lightcurve, and correct ratios and slopes of the synchrotron, $\mathrm{X}$-ray and $\gamma$-ray branches.

\section{The Model: Ingredients and Derivations}

Babadzhanyants and Belokon (1986) were the first to point out, that the appearance of new VLBI knots in the jet of $3 \mathrm{C} 345$ is strictly correlated to a major outburst in the optical. It is therefore natural, to make the knots responsible for the flare emission.

Quasi periodic peaks during the outburst on timescales much longer than acceleration or cooling times of the involved particles, make it necessary to assume a somewhat periodic modulation process.

The "Lighthouse Model" explains the violent variability in Blazars in terms of a modulation of emission enhancement by a variation of the angle between the line of sight and the emission cone of the relativistically moving source.

Since the matter that later forms the jet plasma was accreted in a disk, it carries angular momentum into the jet. Combining the assumption, that the plasma leaves the disk in the very vicinity of the rapidly rotating black 
hole with jet stability calculations from Appl and Camenzind (1993a,b) one obtains the correct timescales for the peak-to-peak periods with a central mass of circa $10^{10} M_{\odot}$ for the most luminous and therefore massive Blazars.

The form of the lightcurve suggests, that the collimated jet opens at some distance from the core, and the emitted flux drops significantly by adiabatic cooling of the radiating particles. Our results are consistent with the assumption, that the opening occurs when the jet leaves the core of the host galaxy, i.e. at a distance of $50-100 p c$ from the black hole (Kormendy 1994).

The variation of the flux is simultaneous in all regimes originating in the moving component.

Comparing the energy densities of the various radiation fields in the core of the AGN's host galaxy yields that in the rest frame of the blob the synchrotron radiation dominates the lower frequencies, while blue shifted and Doppler enhanced ambient, diffuse radiation dominates the higher energies. Calculating the scattering rates following Blumenthal and Gould 1970 off an evolving electron distribution (Kardashev 1962) and transforming back into the observer's frame shows that during the outburst:

- $\mathrm{GHz}$ to optical is dominated by synchrotron emission,

- X-ray is dominated by synchrotron-self-Compton radiation, and

- $\boldsymbol{\gamma}$-ray is made by inverse Compton scattered ambient radiation, all of which originate in the moving component.

A formal treatment on this matter with more details can be found Schramm et al. 1993 and Wagner et al. 1995, the emission mechanisms and energetics are being addressed in Dreissigacker \& Camenzind 1996.

\section{References}

Appl, S. and Camenzind, M.: 1993a, $A \mathscr{B} A$, 270, 71

Appl, S. and Camenzind, M.: 1993b, $A \mathcal{E} A$, 274, 699

Babadzhanyants, M. and Belokon, E.T.: 1986, Astrophysics, 23, 639

Bahcall, J.N., et al.: MNRAS, in press.

Blumenthal, G.R. and Gould, R.J.: 1970, Rev. Mod. Phys., 42.2, 237

Dreissigacker, O. and Camenzind, M.: 1996, in prep.

Kardashev, N.S.: 1962, Sov. Astron., 6, 317.

Schramm, K.J., et al.: 1993, $A \& A, 278,391$

Steffen, W., et al.: $1995 A \& A, 302,335$.

Wagner, S.J. et al.: 1995, $A \& A, 298,688-698$.

Zensus, J.A., et al.: 1995, $A p J, 443,35-53$. 